\title{
Resultados funcionales de las fracturas desplazadas del cuello del radio en niños: correlación entre las medidas de resultado informadas por el paciente (PROMIS y QuickDASH)
}

\author{
Diego Tourn, J. Javier Masquijo \\ Departamento de Ortopedia y Traumatología Infantil, Sanatorio Allende, Córdoba, Argentina
}

\begin{abstract}
RESUMEN
Introducción: Nuestros objetivos fueron: 1) evaluar los resultados subjetivos en pacientes esqueléticamente inmaduros que habían sido sometidos a una cirugía por fracturas desplazadas del cuello del radio, 2) comparar el desempeño del PROMIS y QuickDASH y 3) evaluar los efectos de piso y techo de las medidas de resultado. Materiales y Métodos: Se evaluaron los datos demográficos, las lesiones asociadas, el tipo de fractura, la técnica quirúrgica y las complicaciones. La evaluación funcional se realizó con la escala QuickDASH y el instrumento PROMIS (Upper Extremity/Strength/Pain Interference). Las correlaciones de las escalas se efectuaron con el coeficiente rho de Spearman. El valor alfa se estableció en 0,05. Resultados: Se incluyó a 26 pacientes (13 varones) con una edad promedio de 7.5 años. El seguimiento promedio fue de 31 meses. Hubo correlaciones significativas entre las métricas QuickDASH y PROMIS Upper Extremity posoperatorias $(r s=-0,64 ; p=0,003)$. No se halló una correlación entre PROMIS Pain Interference y PROMIS Strength con el QuickDASH ( $r s=0,001 ; p=0,966$ y $r s=-0,39 ; p=0,101$, respectivamente). Se observaron efectos de techo o piso en todas las escalas. Conclusiones: Hubo una marcada correlación entre el PROMIS Upper Extremity y el QuickDASH para evaluar los resultados después de la cirugía de fracturas del cuello del radio en niños. Sin embargo, todas las medidas tuvieron marcados efectos de piso y techo, probablemente debido al tamaño de la cohorte y a que un alto porcentaje de estas fracturas suelen tener resultados clínicos favorables.
\end{abstract}

Palabras clave: Codo; fractura; cuello del radio; niños, PROMIS.

Nivel de Evidencia: IV

Functional Outcomes of Displaced Radial Neck Fractures in Children: Correlation Between Patient-reported Outcomes (PROMIS and QuickDash)

\begin{abstract}
Introduction: Objectives: 1) to evaluate the subjective outcomes in skeletally immature patients undergoing surgical treatment of displaced radial neck fractures, 2) to compare the performance between PROMIS and QuickDash, 3) to evaluate the floor/ceiling effects of the outcome measurements. We hypothesized that the PROMIS scale would correlate favorably with QuickDash and would demonstrate lower floor or ceiling effects. Materials and Methods: Demographic data, associated lesions, fracture type, surgical technique, and complications were evaluated. QuickDash and PROMIS scales (PROMIS Upper Extremity (UE), PROMIS Strength and PROMIS Pain interference) were used for functional assessment. The Shapiro-Wilk test was used to analyze the normal distribution of the data. Metrics correlations were made with Spearman's rho coefficient. Ceiling and floor effects were further assessed. The alpha value was set at 0.05 . Results: 26 patients were evaluated. Average age: 7.5 years; average followup: 31 months. There were significant correlations between the postoperative QuickDash and PROMIS UE metrics ( $r s=-0.64, p$ $=0.003$ ). We found no correlation between PROMIS Pain and PROMIS Strength with QuickDash ( $r s=0.001, p=0.966$ and $r s=$ $-0.39, p=0.101$ respectively). Ceiling or floor effects were observed at all scales. Conclusion: We observed a strong correlation between PROMIS UE and QuickDash assessing the results after surgical treatment of radial neck fractures in children. However, all the measures had evident floor/ceiling effects, probably due to the size of the cohort and the fact that a high percentage of these fractures usually present favorable clinical outcomes.
\end{abstract}

Keywords: Elbow; radial neck fracture; children; PROMIS.

Level of Evidence: IV

Recibido el 12-10-2020. Aceptado luego de la evaluación el 1-4-2021 • Dr. DIEGO TOURN • diegoatourn@gmail.com ID https://orcid.org/0000-0002-7149-5798

Cómo citar este artículo: Tourn D, Masquijo JJ. Resultados funcionales de las fracturas desplazadas del cuello del radio en niños: correlación entre las medidas de resultado informadas por el paciente (PROMIS y QuickDASH). Rev Asoc Argent Ortop Traumatol 2021;86(5):595-600. https://doi.org/10.15417/issn. 1852-7434.2021.86.5.1276 


\section{INTRODUCCIÓN}

En los niños, las fracturas del cuello del radio representan el 1\% de todas las fracturas pediátricas ${ }^{1}$ y el 5-10\% de las fracturas de codo. ${ }^{2,3}$ Estas fracturas son más frecuentes en niños, porque la cabeza radial es, en gran parte, cartilaginosa hasta que la epífisis proximal comienza a cerrarse alrededor de los 14 o 15 años en los varones y entre los 12 y 14 años en las niñas. ${ }^{4}$ La principal fuerza actuante en estas lesiones se aplica sobre la cabeza del radio y se transmite al cuello, el cual se fractura. Muchas de estas lesiones son consecuencia de una caída sobre el brazo hiperextendido con el codo en extensión asociado a un mecanismo en valgo sobre el antebrazo. 5

El abordaje de una fractura del radio proximal debe ser escalonado desde la reducción cerrada hasta la reducción asistida percutánea y el tratamiento abierto. ${ }^{6}$ La decisión se toma sobre la base de parámetros radiográficos (angulación y traslación) y clínicos (rango de movilidad pronosupinación). Las fracturas con una angulación $<30^{\circ}$ y $<2-3 \mathrm{~mm}$ de traslación pueden tratarse en forma conservadora sin necesidad de reducción, siempre que la pronación y la supinación sean completas. Las fracturas con una angulación $>30^{\circ}$ y traslación $>3 \mathrm{~mm}$ deben tratarse inicialmente con un intento de reducción cerrada bajo sedación o anestesia. Si la reducción cerrada no tiene éxito, se debe intentar la reducción asistida por vía percutánea. Esto se puede realizar manipulando directamente el fragmento con una aguja de Kirschner o un elevador Freer. Otra opción es la técnica de Metaizeau, que utiliza un clavo elástico en forma retrógrada, enganchando el fragmento proximal para su manipulación y fijación, o la combinación de técnicas. ${ }^{7,8}$ Finalmente, las fracturas irreducibles o inestables deben tratarse con reducción abierta y fijación interna.

Múltiples estudios han comunicado los resultados del tratamiento quirúrgico de fracturas desplazadas del cuello del radio en niños. ${ }^{4-8}$ Los resultados funcionales están influenciados por factores, como la edad, el mecanismo de la lesión, las características de la fractura y el tipo de tratamiento. Los resultados percibidos por los pacientes (Patient-Reported Outcomes) han adquirido una creciente importancia en medicina para fundamentar los beneficios de diversos tratamientos. ${ }^{9}$ El instrumento PROMIS (Patient-Reported Outcomes Measurement Information System) fue desarrollado hace algunos años, en los Estados Unidos, por los National Institutes of Health como un medio de estandarizar los puntajes de resultado informados por los pacientes y medir la calidad de vida relacionada con la salud. Estos sistemas de puntaje se han implementado hace poco tiempo, por lo que existen pocos estudios que comuniquen los resultados percibidos por los pacientes sobre los resultados funcionales de la cirugía de fracturas desplazadas del cuello del radio en esta población etaria.

Los objetivos de nuestro estudio fueron: 1) evaluar los resultados subjetivos en pacientes que se habían sometido a una cirugía de fractura desplazada del cuello del radio, 2) comparar el desempeño de los puntajes PROMIS con el del QuickDASH y 3) evaluar los efectos de piso y techo de las medidas de resultado. Nuestra hipótesis es que el instrumento PROMIS se correlacionaría favorablemente con el QuickDASH y demostraría efectos reducidos de piso o techo.

\section{MATERIALES Y MÉTODOS}

\section{Selección de los pacientes}

Luego de obtener la autorización del comité de ética de la institución, se realizó un estudio descriptivo, retrospectivo, en el que se evaluó a todos los pacientes con fractura del cuello del radio entre enero de 2013 y enero de 2019. Todos fueron tratados por cuatro especialistas del Departamento de Traumatología infantil. Los pacientes eran esqueléticamente inmaduros cuando se diagnosticó la fractura. Se excluyó a aquellos que tuvieron un seguimiento $<6$ meses. Se evaluaron los datos demográficos y radiográficos, las complicaciones y las lesiones asociadas. Las complicaciones fueron definidas como cualquier alteración del curso previsto de la respuesta local o sistémica del paciente quirúrgico (infección, consolidación viciosa, seudoartrosis, rigidez, necrosis avascular). Se compararon los tipos de fractura del cuello del radio según la clasificación de Judet, y la técnica de reducción y fijación del fragmento. La evaluación funcional se realizó con el cuestionario QuickDASH ${ }^{10,11}$ y el instrumento PROMIS CAT (PROMIS Upper Extremity, PROMIS Strength y PROMIS Pain Interference). ${ }^{12,13}$ Los pacientes fueron contactados por teléfono, se les informó sobre este estudio y luego de dar su consentimiento, se les enviaron los formularios para ser completados y supervisados. 


\section{Evaluación de las imágenes}

El análisis y almacenamiento de las imágenes se realizaron con el programa de imágenes Kodak Carestream PACS Versión 10.2. Dos observadores evaluaron las imágenes de manera separada y luego definieron las mediciones por consenso. En las radiografías de frente y de perfil de codo, se evaluaron la angulación y la traslación antes del tratamiento y en la primera radiografía tomada después. Se empleó la clasificación de Judet ${ }^{14}$ y, por último, se documentó la consolidación o no de la lesión.

\section{Análisis estadístico}

Se utilizó la prueba de Shapiro-Wilk para analizar la normalidad de los datos. Las diferencias en los valores de angulación y traslación preoperatorios y posoperatorios se evaluaron con pruebas t de Student. Las correlaciones de las escalas se efectuaron mediante el coeficiente rho de Spearman. Se evaluaron, además, los efectos de techo y piso para estas medidas de resultado. El valor alfa se estableció en 0,05 . Todos los análisis estadísticos se realizaron con el programa SPSS v.19.0.

\section{RESULTADOS}

Se operó a 26 pacientes (13 niños) con 26 fracturas. La edad promedio era de 7.5 años (rango 3-15). Según la clasificación de Judet, cinco fracturas eran tipo II; 14, tipo III y siete, tipo IV. El 11\% tenía lesiones asociadas: fractura de cúbito desplazada $(\mathrm{n}=1)$ y fractura de olécranon $(\mathrm{n}=1)$. Once pacientes fueron tratados con reducción cerrada y yeso; cuatro, con enclavijado percutáneo y 11, con enclavado endomedular elástico según la técnica de Metaizeau (Figura).

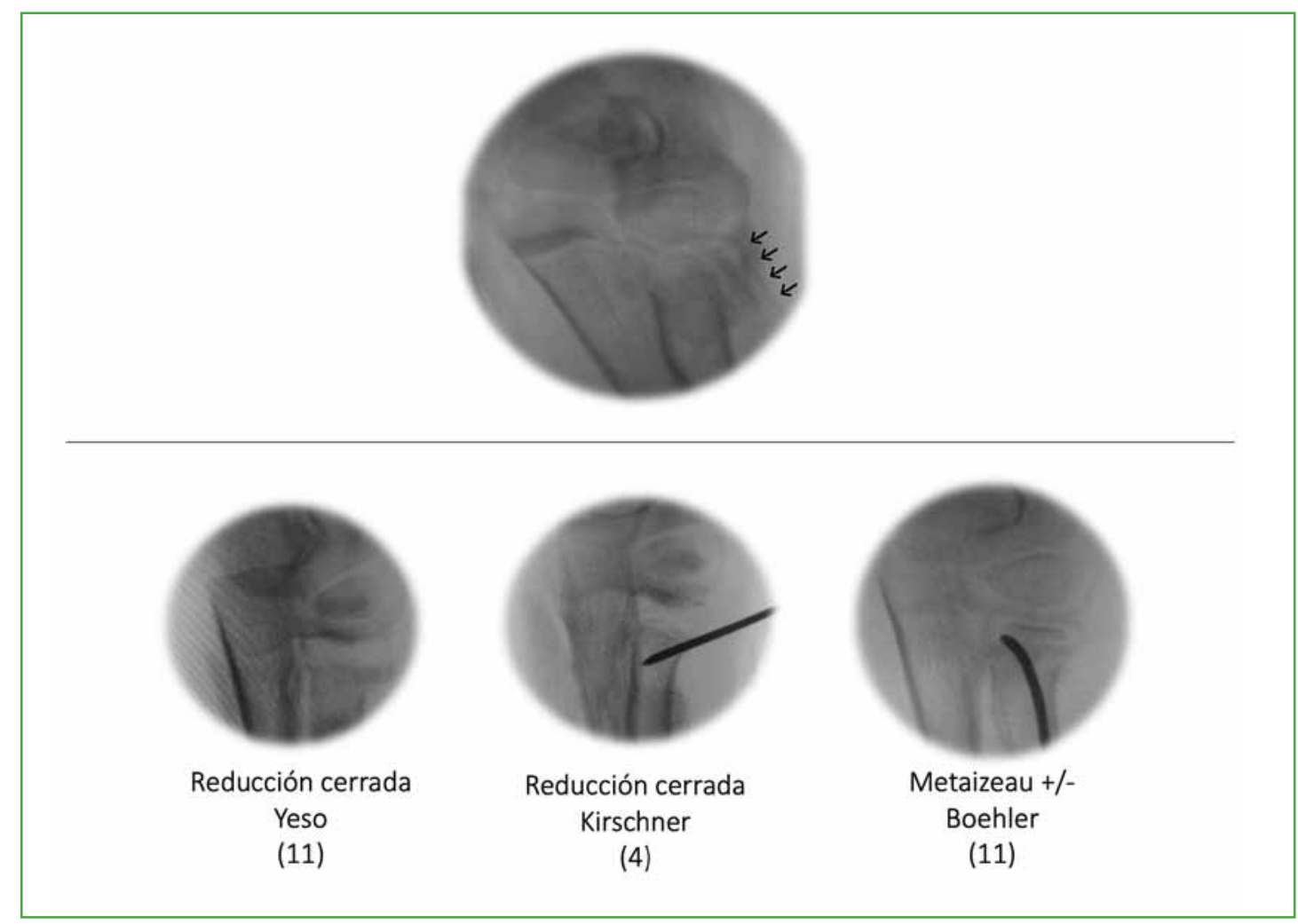

Figura. Distribución de los tratamientos realizados en nuestra serie. 
El seguimiento promedio fue de 31 meses. La angulación y la traslación máximas promedio mejoraron de $47,1^{\circ}$ y $4 \mathrm{~mm}$ antes de la cirugía a $5,5^{\circ}$ y $0,3 \mathrm{~mm}$, respectivamente, en la radiografía posoperatoria inmediata (Tabla 1). Todas las fracturas consolidaron en un promedio de 5.5 semanas (rango 4-6). No hubo complicaciones. Todos los pacientes tenían un rango de movilidad completa en el último seguimiento. Once requirieron una segunda intervención para retirar el material de osteosíntesis (clavo elástico) y dos, una extracción de clavijas por consultorio.

Tabla 1. Valores de angulación y traslación preoperatorios y posoperatorios

\begin{tabular}{|l|c|c|c|}
\hline & Preoperatorio & Posoperatorio & $\mathbf{p}^{*}$ \\
\hline Angulación & $47,05^{\circ}$ & $5,5^{\circ}$ & $<0,01$ \\
\hline Traslación $(\mathrm{mm})$ & 4 & 0 & $<0,01$ \\
\hline
\end{tabular}

Diecinueve pacientes pudieron completar las escalas funcionales y se los pudo evaluar. Hubo correlaciones significativas entre las métricas QuickDASH y PROMIS Upper Extremity posoperatorias ( $r s=-0,64 ; p=0,003$ ). No se halló una correlación entre las métricas PROMIS Pain Interference y PROMIS Strength con el puntaje QuickDASH ( $r s=0,001 ; p=0,966$ y rs $=-0,39 ; p=0,101$, respectivamente). Se observaron efectos de techo o piso en todas las escalas (Tablas 2 y 3 ).

Tabla 2. Resultados reportados por los pacientes

\begin{tabular}{|l|c|c|c|c|c|}
\hline Escala & $\begin{array}{c}\text { Total de } \\
\text { pacientes }\end{array}$ & Media & $\begin{array}{c}\text { Desviación } \\
\text { estándar }\end{array}$ & Mánimo \\
\hline PROMIS Upper Extremity & 19 & 37,44 & 7,03 & 10 & 40 \\
\hline PROMIS Pain Interference & 19 & 13,95 & 2,41 & 13 & 22 \\
\hline PROMIS Strength & 19 & 30,13 & 10,9 & 8 & 40 \\
\hline QuickDASH & 19 & 5,94 & 9,28 & 0 & 38,64 \\
\hline
\end{tabular}

PROMIS Upper Extremity vs. QuickDASH: $r s=-0,63918, \mathrm{p}$ (de dos colas) $=0,00322$

PROMIS Pain Interference vs. QuickDASH: rs $=0,01047, \mathrm{p}$ (de dos colas) $=0,96608$

PROMIS Strength vs. QuickDASH: $r s=-0,38728, p($ de dos colas $)=0,10139$

Tabla 3. Efecto techo/piso

\begin{tabular}{|l|c|c|c|}
\hline Escala & n & Efecto & $\begin{array}{c}\text { Número total de escalas } \\
\text { en valores máximo/mínimo }(\%)\end{array}$ \\
\hline PROMIS Upper Extremity & 19 & Techo & $14(73 \%)$ \\
\hline PROMIS Pain Interference & 19 & Piso & $15(78 \%)$ \\
\hline PROMIS Strength & 19 & Techo & $8(42 \%)$ \\
\hline QuickDASH & 19 & Piso & $9(47 \%)$ \\
\hline
\end{tabular}




\section{DISCUSIÓN}

Las medidas de resultado informadas por el paciente han ganado popularidad en los últimos años tanto en la atención clínica como en la investigación en cirugía ortopédica. En la práctica clínica pediátrica, hay múltiples ejemplos validados y de uso frecuente, como el PODCI (Pediatric Outcomes Data Collection Instrument), el CHQ (Child Health Questionnaire), el PedsQL (Pediatric Quality of Life Inventory). Todas estas medidas de resultado contienen dominios de función física y no hay consenso sobre cuál sería superior. El instrumento PROMIS no se utiliza para una enfermedad específica, lo que mejora su aplicabilidad y lo posiciona como una posible medida de resultado universal. Se ha validado en poblaciones de pacientes con trastornos ortopédicos de pie y tobillo, extremidad superior y columna. ${ }^{15-18}$ Las medidas de función física del PROMIS han demostrado ser útiles para evaluar los resultados ortopédicos y son superiores a las medidas históricas en varias poblaciones. A pesar de que el PROMIS ha comenzado a utilizarse en diversas áreas de la ortopedia general, existen escasos estudios que evalúen su utilidad en pacientes pediátricos. En esta serie, en la cual analizamos un grupo específico de fracturas pediátricas del miembro superior, observamos una correlación significativa entre las métricas QuickDASH y PROMIS Upper Extremity posoperatorias, aunque no se halló una correlación entre las métricas PROMIS Pain Interference y PROMIS Strength con el puntaje QuickDASH. Esto podría deberse a diversos factores, como el tamaño de la muestra y el pronóstico favorable de las fracturas evaluadas. Por otra parte, en todos los casos, se presentaron efectos piso y techo. Esto podría explicarse porque, en el período evaluado, solo el 15\% de nuestra cohorte era $>10$ años y, en ningún caso, se requirió una reducción abierta. Estudios previos han demostrado que los resultados son más favorables cuando se utilizan técnicas de reducción cerrada o percutáneas y cuando la fractura ocurre en niños $<10$ años. ${ }^{19,20}$

Este estudio presenta ciertas limitaciones debido a su diseño metodológico y al tamaño relativamente pequeño de la muestra. Si bien el puntaje QuickDASH ha sido validado en español y utilizado previamente en estudios que incluyen pacientes $<18$ años, ${ }^{21,22}$ es una escala diseñada originalmente para adultos. Por contrapartida, el instrumento PROMIS ha sido diseñado para la población pediátrica, aunque no ha sido validado en español. En nuestro estudio, la edad promedio era de 7 años, por lo que la anamnesis fue indirecta en la mayoría de los pacientes incluidos. En la bibliografía, está bien documentado, tanto para adultos como para niños, que la información proporcionada por anamnesis indirecta no es equivalente a la informada por el paciente. ${ }^{23} \mathrm{La}$ varianza entre informantes (falta de concordancia entre el autoinforme y el informe sustitutivo) podría afectar los resultados obtenidos en nuestro estudio, ${ }^{24,25}$ principalmente en los dominios de fuerza y dolor.

En conclusión, hallamos una marcada correlación entre las métricas PROMIS Upper Extremity y QuickDASH para evaluar los resultados después de la cirugía de fractura del cuello del radio en niños. Sin embargo, todas las medidas presentaron marcados efectos de piso y techo, probablemente debido al tamaño de la cohorte y a que un alto porcentaje de estas fracturas suelen tener resultados clínicos favorables. Estudios futuros nos permitirán evaluar la utilidad de estas medidas de resultado en otras enfermedades de los niños.

Conflicto de intereses: Los autores no declaran conflictos de intereses.

\section{BIBLIOGRAFÍA}

1. O’Brien PI. Injuries involving radial head epiphysis. Clin Orthop 1965;41:51-8. PMID: 5832738

2. Gutiérrez-de la Iglesia D, Pérez-López LM, Cabrera-González M, Knörr-Giménez J. Surgical techniques for displaced radial neck fractures: predictive factors of functional results. J Pediatr Orthop 2017;37(3):159-65. https://doi.org/10.1097/BPO.0000000000000617

3. Skaggs DL, Mirzayan R. The posterior fat pad sign in association with occult fracture of the elbow in children. $J$ Bone Joint Surg Am 1999;81(10):1429-33. https://doi.org/10.2106/00004623-199910000-00007

4. Ackerson R, Nguyen A, Carry PM, Pritchard B, Hadley-Miller N, Scott F. Intra-articular radial head fractures in the skeletally immature patient: complications and management. J Pediatr Orthop 2015;35(5):443-8. https://doi.org/10.1097/BPO.0000000000000302 
5. De Mattos CB, Ramski DE, Kushare IV, Angsanuntsukh C, Flynn JM. Radial neck fractures in children and adolescents: an examination of operative and nonoperative treatment and outcomes. J Pediatr Orthop 2016;36(1):612. https://doi.org/10.1097/BPO.0000000000000387

6. Nicholson LT, Skaggs DL. Proximal radius fractures in children. J Am Acad Orthop Surg 2019;27(19):e876-e886. https://doi.org/10.5435/JAAOS-D-18-00204

7. Pring ME. Pediatric radial neck fractures: when and how to fix. J Pediatr Orthop 2012;32(Suppl 1):S14-21. https://doi.org/10.1097/BPO.0b013e31824b251d

8. Wang J, Chen W, Guo M, Su Y, Zhang Y. Percutaneous reduction and intramedullary fixation technique for displaced pediatric radial neck fractures. J Pediatr Orthop B 2013;22(2):127-32. https://doi.org/10.1097/BPB.0b013e32835b5700

9. Gerull WD, Okoroafor UC, Guattery J, Goldfarb CA, Wall LB, Calfee RP. Performance of Pediatric PROMIS CATs in children with upper extremity fractures. Hand (NY) 2020;15(2):194-200. https://doi.org/10.1177/1558944718793195

10. Beaton DE, Wright JG, Katz JN; Upper Extremity Collaborative Group. Development of the QuickDASH: comparison of three item-reduction approaches. J Bone Joint Surg Am 2005;87(5):1038-46. https://doi.org/10.2106/JBJS.D.02060

11. Hudak PL, Amadio PC, Bombardier C. Development of an upper extremity outcome measure: the DASH (disabilities of the arm, shoulder and hand) [corrected]. The Upper Extremity Collaborative Group (UECG). Am J Ind Med 1996;29(6):602-8. Erratum en: Am J Ind Med 1996;30(3):372. https://doi.org/10.1002/(SICI)1097-0274(199606)29:6<602::AID-AJIM4>3.0.CO;2-L

12. DeWalt DA, Rothrock N, Yount S, Stone AA; PROMIS Cooperative Group. Evaluation of item candidates: the PROMIS qualitative item review. Med Care 2007;45(5 Suppl 1):S12-21. https://doi.org/10.1097/01.mlr.0000254567.79743.e2

13. Brodke DJ, Saltzman CL, Brodke DS. PROMIS for orthopaedic outcomes measurement. J Am Acad Orthop Surg 2016;24(11):744-9. https://doi.org/10.5435/JAAOS-D-15-00404

14. Judet J, Judet R, Lefranc J. Fracture du col radial chez l'enfant. Ann Chir 1962;16:1377-85. PMID: 13957959

15. Hung M, Baumhauer JF, Latt LD, Saltzman CL, SooHoo NF, Hunt KJ; National Orthopaedic Foot \& Ankle Outcomes Research Network. Validation of PROMIS® Physical Function computerized adaptive tests for orthopaedic foot and ankle outcome research. Clin Orthop Relat Res 2013;471(11):3466-74. https://doi.org/10.1007/s11999-013-3097-1

16. Hung M, Clegg DO, Greene T, Saltzman CL. Evaluation of the PROMIS physical function item bank in orthopaedic patients. J Orthop Res 2011;29(6):947-53. https://doi.org/10.1002/jor.21308

17. Hung M, Hon SD, Franklin JD, Kendall RW, Lawrence BD, Neese A, et al. Psychometric properties of the PROMIS physical function item bank in patients with spinal disorders. Spine (Phila Pa 1976) 2014;39(2):158-63. https://doi.org/10.1097/BRS.0000000000000097

18. Overbeek CL, Nota SP, Jayakumar P, Hageman MG, Ring D. The PROMIS physical function correlates with the QuickDASH in patients with upper extremity illness. Clin Orthop Relat Res 2015;473(1):311-7. https://doi.org/10.1007/s11999-014-3840-2

19. Basmajian HG, Choi PD, Huh K, Sankar WN, Wells L, Arkader A. Radial neck fractures in children: experience from two level-1 trauma centers. J Pediatr Orthop B 2014;23(4):369-74. https://doi.org/10.1097/BPB.0000000000000057

20. Zimmerman RM, Kalish LA, Hresko MT, Waters PM, Bae DS. Surgical management of pediatric radial neck fractures. J Bone Joint Surg Am 2013;95(20):1825-32. https://doi.org/10.2106/JBJS.L.01130

21. Canavese F, Athlani L, Marengo L, Rousset M, Rouel-Rabiau N, Samba A, et al. Evaluation of upper-extremity function following surgical treatment of displaced proximal humerus fractures in children. J Pediatr Orthop B 2014;23(2):144-9. https://doi.org/10.1097/BPB.0000000000000009

22. Bae DS, Gholson JJ, Zurakowski D, Waters PM. Functional outcomes after treatment of scaphoid fractures in children and adolescents. J Pediatr Orthop 2016;36(1):13-8. https://doi.org/10.1097/BPO.0000000000000406

23. Sprangers MA, Aaronson NK. The role of health care providers and significant others in evaluating the quality of life of patients with chronic disease: a review. J Clin Epidemiol 1992;45(7):743-60. https://doi.org/10.1016/0895-4356(92)90052-o

24. Achenbach TM, McConaughy SH, Howell CT. Child/adolescent behavioral and emotional problems: implications of cross-informant correlations for situational specificity. Psychol Bull 1987;101(2):213-32. PMID: 3562706

25. Upton P, Lawford J, Eiser C. Parent-child agreement across child health-related quality of life instruments: a review of the literature. Qual Life Res 2008;17(6):895-913. https://doi.org/10.1007/s11136-008-9350-5 